\title{
Double Exchange Alone Does Not Explain the Resistivity of $\mathrm{La}_{1-\mathrm{x}} \mathrm{Sr}_{\mathrm{x}} \mathrm{MnO}_{3}$
}

\author{
A. J. Millis \\ P. B. Littlewood \\ B. I. Shraiman \\ ATET Bell Laboratories \\ Murray Hill, NJ 07974
}

\begin{abstract}
The $\mathrm{La}_{1-\mathrm{x}} \mathrm{Sr}_{\mathrm{x}} \mathrm{MnO}_{3}$ system with $0.2 \lesssim \mathrm{x} \lesssim 0.4$ has traditionally been modelled with a "double exchange" Hamiltonian, in which it is assumed that the only relevant physics is the tendency of carrier hopping to line up neighboring spins. We present a solution of the double exchange model, show it is incompatible with many aspects of the resistivity data, and propose that a strong electron-phonon interaction arising from a Jahn-Teller splitting of the outer Mn d-level plays a crucial role.
\end{abstract}

Typeset using REVTEX 
The $\mathrm{La}_{1-\mathrm{x}} \mathrm{A}_{\mathrm{x}} \mathrm{MnO}_{3}$ system (A represents a divalent alkali element such as $\mathrm{Sr}$ or Ca) has attracted much recent attention because of the very large magnetoresistance exhibited for $0.2 \lesssim \mathrm{x} \lesssim 0.4$ [1]. Treatments [2 [4] of the physics of this system have focussed primarily on the phenomenon of "double exchange" [5]. In this communication we show that a straightforward and reasonably complete solution of the double exchange model is possible. From this solution we calculate the frequency and temperature dependence of the conductivity and show that it disagrees in several important respects with the experimental data. The discrepancy must be resolved by including additional physics, which we suggest involves polaron effects due to a very strong electron-phonon coupling coming from a Jahn-Teller splitting of the $\mathrm{Mn}^{3+}$ ion. We mention several experiments which have apparently not yet been performed and which would verify or falsify our suggestion.

In $\mathrm{La}_{1-\mathrm{x}} \mathrm{A}_{\mathrm{x}} \mathrm{MnO}_{3}$ the electronically active orbitals are the $\mathrm{Mn}_{\mathrm{x}^{2}-\mathrm{y}^{2}}$ and $\mathrm{d}_{3 \mathrm{z}^{2}-\mathrm{r}^{2}}$ orbitals [6]. The mean number of d-electrons per Mn is 4-x, the Hunds rule coupling is believed to be very strong relative to the $\mathrm{d}$-d hopping and the spin-orbit coupling [7] so the spins of all of the d-electrons on a given site must be parallel. Three of the d electrons go into tightly bound core-like $d_{x y}, d_{x z}, d_{y z}$ orbitals forming a core spin $S_{i}^{c}$ of magnitude $3 / 2$, to which the outer shell electron (which may hop from site to site) is aligned by the Hund's rule coupling. The Hamiltonian containing this physics is

$$
\mathrm{H}_{\mathrm{d}-\mathrm{ex}}=-\sum_{\langle\mathrm{ij}\rangle \mathrm{a}, \mathrm{b}, \alpha} \mathrm{t}_{\mathrm{ij}}^{\mathrm{ab}} \mathrm{d}_{\mathrm{ia} \alpha}^{\dagger} \mathrm{d}_{\mathrm{j} b \alpha}-\mathrm{J}_{\mathrm{H}} \sum_{\mathrm{i} \alpha \beta} \overrightarrow{\mathrm{S}}_{\mathrm{i}}^{\mathrm{c}} \cdot \mathrm{d}_{\mathrm{i} \alpha \alpha}^{\dagger} \vec{\sigma}_{\alpha \beta} \mathrm{d}_{\mathrm{i} a}
$$

Here $\mathrm{d}_{\mathrm{i} \alpha \alpha}^{\dagger}$ creates an electron in an outer-shell orbital state $\mathrm{a}=\mathrm{x}^{2}-\mathrm{y}^{2}$ or $3 \mathrm{z}^{2}-\mathrm{r}^{2}$ and spin $\alpha, \mathrm{J}_{\mathrm{H}}$ is the Hunds rule coupling connecting the core spin to the outer shell electrons, and the interesting limit, which we shall take, is $\mathrm{J}_{\mathrm{H}} \rightarrow \infty$. To study Eq. (1) it is convenient to parametrize $\mathrm{S}_{\mathrm{i}}^{\mathrm{c}}$ by polar angles $\theta_{\mathrm{i}}, \phi_{\mathrm{i}}$ and to rotate the electrons so that the spin quantization axis at, site $\mathrm{i}$ is parallel to $\mathrm{S}_{\mathrm{i}}^{\mathrm{c}}$ on site $\mathrm{i}$ and then project on to the component parallel to $\overrightarrow{\mathrm{S}_{\mathrm{i}}^{c}}$. The matrix $\mathbf{R}_{\mathrm{i}}$ which accomplishes this is $\mathbf{R}_{\mathrm{i}}=\cos \theta_{\mathrm{i}} / 2$ $\boldsymbol{1}+\operatorname{isin}\left(\theta_{\mathrm{i}} / 2\right) \sin \phi_{\mathrm{i}} \boldsymbol{\sigma}^{\mathrm{x}}+\operatorname{isin}\left(\theta_{\mathrm{i}} / 2\right) \cos \phi_{\mathrm{i}} \boldsymbol{\sigma}^{\mathrm{y}}$. The electrons may be integrated out, and 
the partition function $\mathrm{Z}$ written as

$$
\mathrm{Z}=\int \mathcal{D} \cos \theta \mathcal{D} \phi_{\mathrm{i}} \exp \mathcal{A}
$$

with the action $\mathcal{A}$ given by

$$
\begin{array}{r}
\mathcal{A}=\operatorname{Tr} \ln \left[\partial_{\tau}-\mu+\mathrm{R}_{\mathrm{i}}^{+} \partial_{\tau} \mathrm{R}_{\mathrm{i}}-\mathrm{t}_{\mathrm{ij}}^{\mathrm{ab}}\left(\mathrm{R}_{\mathrm{i}}^{\dagger} \mathrm{R}_{\mathrm{j}}+\mathrm{R}_{\mathrm{J}}^{\dagger} \mathrm{R}_{\mathrm{i}}\right)\right]_{11} \\
+\mathrm{i} \mathrm{S}_{\mathrm{c}} \int_{0}^{\mathrm{B}} \mathrm{d} \tau \sum_{\mathrm{i}} \dot{\phi}_{\mathrm{i}}\left(1-\cos \theta_{\mathrm{i}}\right)
\end{array}
$$

Here the first term comes from integrating out the electrons and the second is the Berry phase term for the core spins. The subscript 11 on the argument of the logarithm comes from the requirement that in the $\mathrm{J}_{\mathrm{H}} \rightarrow \infty$ limit the outer shell electron must be parallel to $\vec{S}_{\mathrm{c}}$. At low $\mathrm{T}$ we may expand about the ordered ferromagnetic state $\theta_{\mathrm{i}}=0$. The effective action becomes $\mathcal{A}=\mathcal{A}_{\mathrm{F}}+\mathcal{A}_{\mathrm{sw}} \cdot \mathcal{A}_{\mathrm{F}}$ is the free energy for free fermions moving in the band structure defined by $\mathrm{t}_{\mathrm{ij}}^{\mathrm{ab}} \cdot \mathcal{A}_{\mathrm{sw}}$ may be written in terms of the magnetization variables $M_{x}$ and $M_{y}$ describing deviations from the ordered state (with magnetization taken parallel to z) as

$$
\begin{aligned}
\mathcal{A}_{\mathrm{sw}} & =\frac{1}{2} \int_{0}^{\mathrm{B}} \mathrm{d} \tau \int \frac{\mathrm{d}^{3} \mathrm{k}}{(2 \pi)^{3}} \mathrm{i}\left(\mathrm{S}_{\mathrm{c}}+\frac{1-\mathrm{x}}{2}\right) \overrightarrow{\mathrm{M}}_{\mathrm{k}} \times \partial_{\tau} \overrightarrow{\mathrm{M}}_{-\mathrm{k}} \\
& -2 \mathrm{Ka}^{2} \mathrm{k}^{2} \overrightarrow{\mathrm{M}}_{\mathrm{k}} \cdot \overrightarrow{\mathrm{M}}_{-\mathrm{k}}+\mathcal{O}\left(\mathrm{M}^{4}\right)
\end{aligned}
$$

Here $\mathrm{K}=\sum_{\mathrm{ab}} \mathrm{t}_{\mathrm{i}, \mathrm{i}+\hat{\mathrm{x}}}^{\mathrm{ab}}\left\langle\mathrm{c}_{\mathrm{ia}}^{\dagger} \mathrm{c}_{\mathrm{i}+\hat{\mathrm{x}} \mathrm{b}}\right\rangle$ is the electron stress- energy tensor and is related to the integral of the optical conductivity as described below. a is the lattice constant. Here $\mathrm{c}_{\mathrm{i}}^{\dagger}$ creates a spin polarized electron on site i. Equation (4) is the action for a quantum ferromagnet with spin $\mathrm{S}^{*}=\mathrm{S}_{\mathrm{c}}+\frac{1-\mathrm{x}}{2}$ and stiffness $\mathrm{K}$. This action implies that the magnon dispersion is

$$
\omega_{\mathrm{mag}}=\left(\mathrm{K} / \mathrm{S}^{*}\right)(\mathrm{ka})^{2}
$$

A very similar result for the magnon dispersion was obtained by Kubo and Ohata [3] using different methods.

The quantity $\mathrm{K}$ is very important because it is the only energy scale in the theory. We may estimate $\mathrm{K} \sim 2 \mathrm{tn}$, where $\mathrm{n}$ is the electron density and the hopping energy. 
A recent band theory calculation found a bandwidth of $2 \mathrm{eV}$ implying $\mathrm{t} \sim 0.25 \mathrm{eV}$ and $\mathrm{K} \sim 0.1 \mathrm{eV}$ for $\mathrm{n}=0.3$. [8].From $\mathrm{K}$ we may, e.g. estimate the ferromagnetic transition temperature as follows: the cubic lattice Heisenberg model with exchange constant $\mathrm{J}$ has a magnon dispersion $\omega=2 \mathrm{JS}(\mathrm{ka})^{2}$ [9]. The known relation [10] between $\mathrm{J}$ and $\mathrm{T}_{\mathrm{c}}$ then implies a $\mathrm{T}_{\mathrm{c}} \approx 2.9 \mathrm{~K}\left(\mathrm{~S}^{*}+1\right) / \mathrm{S}^{*} \approx .3 \mathrm{eV}$, more than an order of magnitude higher than the observed value. This discrepancy, we believe, is evidence that additional physics, not included in the double exchange model, is important for $\mathrm{La}_{1-\mathrm{x}} \mathrm{Sr}_{\mathrm{x}} \mathrm{MnO}_{3}$.

A direct measurement of the magnon spectrum would determine $\mathrm{K}$. In the absence of this measurement, one may estimate K from the optical conductivity $\sigma(\omega)$. In a one-band model with only nearest neighbor hopping $\int_{0}^{\infty} \mathrm{d} \omega \sigma(\omega)=\pi \mathrm{e}^{2} \mathrm{~K} / \mathrm{a}[11$. To extract $\mathrm{K}$ from conductivity data on a real material one must remove the interband contributions to $\sigma$. Ambiguities arise because there is often no clear demarcation between interband and intraband contributions. The optical conductivity of $\mathrm{La}_{1-\mathrm{x}} \mathrm{Sr}_{\mathrm{x}} \mathrm{MnO}_{3}$ has been measured for $\mathrm{x}=0.175$ and $\mathrm{x}=0.3$ [12]. Roughly, $\sigma(\omega) \approx(500 \mu \Omega-\mathrm{cm})^{-1} \approx 0.3 \mathrm{eV}$, independent of $\omega$ and it seems reasonable to assume that for $\omega<1 \mathrm{eV}$ the conductivity is dominated by the conduction band. Using $\mathrm{a}=4 \AA$, one finds $\mathrm{K} \approx 0.03 \mathrm{eV} \approx 400 \mathrm{~K}$, much less than the band structure estimate. Note that even this value of $\mathrm{K}$ implies a magnetic transition temperature. much higher than the observed $\mathrm{T}_{\mathrm{c}} \approx 200 \mathrm{~K}$ for $\mathrm{x}=0.175$.

We now turn to the properties of the model in the regime $\mathrm{T} \sim \mathrm{T}_{\mathrm{c}}$. Because we have already shown that the low $\mathrm{T}$ properties are those of a quantum model with the relatively large spin value $S \approx 2$, it seems reasonable to suppose that near $\mathrm{T}_{\mathrm{c}}$ we may consider classical spins and so neglect the imaginary time dependence of the angular variables in Eq. (3). The problem then becomes that of electrons of electrons moving on a lattice with hopping amplitude $\bar{t}_{\mathrm{ij}}^{\mathrm{ab}}=\mathrm{t}_{\mathrm{ij}}^{\mathrm{ab}}\left[\cos \left(\theta_{\mathrm{i}} / 2\right) \cos \left(\theta_{\mathrm{j}} / 2\right)\right.$ $\left.+\cos \left(\phi_{\mathrm{i}}-\phi_{\mathrm{j}}\right) \sin \left(\theta_{\mathrm{i}} / 2\right) \sin \left(\theta_{\mathrm{j}} / 2\right)\right]$. We further assume that contributions to the partition function in which fermions move on closed loops in real space may be neglected. We may then rotate the $\phi_{\mathrm{i}}$ independently and therefore replace this by the familiar double 
exchange form $\overline{\mathrm{t}}_{\mathrm{ij}}^{\mathrm{ab}}=\mathrm{t}_{\mathrm{ij}}^{\mathrm{ab}} \sqrt{\frac{\mathrm{S}^{2}+\overrightarrow{\mathrm{S}}_{\mathrm{i}} \cdot \overrightarrow{\mathrm{S}}_{\mathrm{j}}}{2 \mathrm{~S}^{2}}}[$ [0]. We may then replace Eq. (1) by

$$
H_{e f f}=-\sum_{i j a b} \frac{t_{i j}^{a b}}{\sqrt{2}} \sqrt{1+\frac{\vec{S}_{i} \cdot \vec{S}_{j}}{S^{2}}}\left(c_{i a}^{+} c_{j b}+\text { h.c. }\right)
$$

where the $\vec{S}_{\mathrm{i}}$ are now understood to be classical spins. The free energy function describing the spin distribution is to be obtained by integrating out the conduction electrons. For any fixed distribution of spins the problem is one of conduction electrons moving in a lattice with random hopping. To a good approximation, the free energy of the conduction electrons depends only on the average hopping [13] so the spin energy $E\left(\left\{S_{i}\right\}\right)$ is given by

$$
\mathrm{E}\left(\left\{\mathrm{S}_{\mathrm{i}}\right\}\right)=-\mathrm{T} \sum_{\mathrm{k}} \ln \left[1+\mathrm{e}^{\beta\left(\epsilon_{\mathrm{k}}-\mu\right)}\right]
$$

with

$$
\bar{\epsilon}_{\mathrm{k}}=-2 \overline{\mathrm{t}}\left(\operatorname{cosk}_{\mathrm{x}} \mathrm{a}+\operatorname{cosk}_{\mathrm{y}} \mathrm{a}+\operatorname{cosk}_{\mathrm{z}} \mathrm{a}\right)
$$

and

$$
\bar{t}=\left\langle t_{i j}^{a b} \sqrt{1+\frac{S_{i} \cdot S_{j}}{S^{2}}}\right\rangle
$$

In particular, if the temperature is less than the Fermi temperature of the electrons,

$$
E\left(\left\{S_{i}\right\}\right)=-\sum_{\langle i j\rangle} \frac{t_{i j}^{a b}}{\sqrt{2}} \sqrt{1+\frac{S_{i} \cdot S_{j}}{S^{2}}}\left\langle c_{i a}^{+} c_{j b}\right\rangle
$$

In other words, the spin energy involves nearest neighbor coupling with scale again set by the electron kinetic energy. In the nearest neighbor Heisenberg model at $\mathrm{T}_{\mathrm{c}},\left\langle\overrightarrow{\mathrm{S}}_{\mathrm{i}} \cdot \overrightarrow{\mathrm{S}}_{\mathrm{j}}\right\rangle / \mathrm{S}^{2} \approx 1 / 3$ [10, so an expansion in $\mathrm{S}_{\mathrm{i}} \cdot \mathrm{S} / \mathrm{S}^{2}$ is reasonable, and we may conclude that the spin energy is given by the nearest neighbor Heisenberg model, with $\mathrm{J}=\mathrm{K} / 2 \sqrt{2}$. Thus thermal effects do not significantly change our estimates of the energy scales, which are much too large to explain the observed transition temperature. 
We now turn to the resistivity of the model near $T_{c}$. Before presenting the details of the calculations, we make some general comments. The electron-spin-fluctuation interaction in Eq. (6) leads to an electron self-energy with real and imaginary parts. The real part leads to a contribution to the electron velocity which increases as the temperature decreases and expresses the physics that as $\left\langle\vec{S}_{\mathrm{i}} \cdot \overrightarrow{\mathrm{S}}_{\mathrm{j}}\right\rangle$ increases, so does the electron hopping. The imaginary part leads to scattering, due physically to fluctuations in $\vec{S}_{\mathrm{i}} \cdot \overrightarrow{\mathrm{S}}_{\mathrm{j}}$. If the spins are treated classically, this scattering is mathematically identical to conventional impurity scattering, and leads to a resistivity proportional to $\left(\mathrm{p}_{\mathrm{F}} \ell\right)^{-1}$ where $\mathrm{p}_{\mathrm{F}}$ is the electron Fermi wavevector and $\ell$ is the mean free path. The mean free path in this static spin approximation is a purely geometric property determined by the amplitude and spatial correlations of the fluctuations in $\vec{S}_{i} \cdot \vec{S}_{j}$ and in particular is independent of the electron velocity. Therefore to compute the resistivity it suffices to calculate the scattering of the electrons off the spin fluctuations, neglecting the possibly large velocity renormalization. Further, we shall see that the scattering is sufficiently weak that the Born approximation suffices. Finally, we note that because the scattering is static the optical conductivity must have essentially the Drude form.

There is no rigorous expression for the dc resistivity. To obtain a reasonable approximate expression we use the convenient and at least qualitatively accurate "memory function" method [14 in which one defines the memory function $\mathrm{M}(\omega, \mathrm{T})$ via

$$
\mathrm{M}(\omega, \mathrm{T})=\int_{0}^{\infty} \mathrm{dt} \mathrm{e}^{\mathrm{i} \omega \mathrm{t}}\left\langle[\mathrm{H}, \mathrm{j}]_{\mathrm{t}},[\mathrm{H}, \mathrm{j}]_{0}\right\rangle
$$

where the current operator $\mathrm{j}$ is

$$
j=i \sum_{i j a b} t_{i j}^{a b}\left(c_{i a}^{+} c_{j b}-c_{j b}^{+} c_{i a}\right)\left(1+\operatorname{frac}_{i} \cdot S_{j} S^{2}\right)
$$

and $\mathrm{H}$ is given by Eq. (6). The Heisenberg representation is assumed and the subscript on the commutator denotes the time argument of the operators. The memory 
function rigorously determines the leading term in a high-frequency expansion of the conductivity. By assuming that this leading term is the first term of an expansion of $\sigma(\omega, \mathrm{T})=\mathrm{Ke}^{2} / \mathrm{a}\left[\mathrm{i} \omega+\frac{\mathrm{M}(\omega, \mathrm{T})}{\mathrm{K}}\right]$ one finds [14 that the temperature dependent resistivity $\rho(\mathrm{T})=\mathrm{e}^{2} \mathrm{M}(\omega=0, \mathrm{~T})$. We have evaluated $\mathrm{M}(\omega=0, \mathrm{~T})$ from Eqs. 6 and 11 to leading order in $1 / \mathrm{S}$ and $\mathrm{k}_{\mathrm{F}} \mathrm{a}$. We find

$$
\rho(\mathrm{T})=\mathrm{e}^{2} \sum_{\mathrm{R}, \delta_{1}, \delta_{2}}\left\langle\overrightarrow{\mathrm{S}}(0) \cdot \overrightarrow{\mathrm{S}}\left(-\vec{\delta}_{1}\right) \overrightarrow{\mathrm{S}}(\mathrm{R}) \cdot \overrightarrow{\mathrm{S}}\left(\mathrm{R}+\delta_{2}\right)\right\rangle / \mathrm{S}^{4} \mathrm{~B}(\mathrm{R})
$$

Here $\vec{R}$ labels sites on the cubic lattice, and $\delta_{1}$ and $\delta_{2}$ are any of the vectors $\hat{x}, \hat{y}, \hat{z}$ connecting a site to one of the nearest neighbors. B is proportional to the electron current-current correlation function weighted by a factor accounting for the ineffectiveness of small q scattering in degrading the current. In the free electron approximation in which the fermions have a $\mathrm{k}^{2}$ dispersion and $\mathrm{t}^{\mathrm{a}}=\mathrm{t}^{\mathrm{b}}=\mathrm{t}$,

$$
B(R)=\frac{9}{32\left(p_{F} a\right)^{4}}\left[\frac{\sin ^{2} p_{F}(\vec{R}+\hat{x})}{\left(p_{F}|\vec{R}+\hat{x}|\right)^{2}}+\frac{\sin ^{2} p_{F}|\vec{R}-\hat{x}|}{\left(p_{F}|R-\hat{x}|\right)^{2}}-\frac{2 \sin ^{2} p_{F} R}{\left(p_{F} R\right)^{2}}\right]
$$

Here $\mathrm{p}_{\mathrm{F}}$ is the Fermi wavevector.

It is interesting to compare Eqs. 11 and 12 to the expression for $\rho(\mathrm{T})$ given by Langer and Fisher [15] who considered the general question of resistive anomalies at magnetic critical points. They began from a model in which the carrier-spin coupling was $\mathrm{H}_{\mathrm{c}-=\mathrm{s}} \sum_{\mathrm{i}} \overrightarrow{\mathrm{S}}_{\mathrm{i}} \cdot \vec{\sigma}_{\mathrm{ci}}$ where $\mathrm{S}_{\mathrm{i}}$ is a local moment and $\sigma_{\mathrm{c}}$ the carrier spin density at site i, and obtained a formula rather similar to Eqs. 11, 12 except that instead of the four spin correlator they obtained simply $\langle\vec{S}(0) \cdot \vec{S}(R)\rangle$ because in their model, local fluctuations of $\mathrm{S}_{\mathrm{i}}$ scatter the electrons while in the model defined by Eq. 6 local fluctuations of $\mathrm{S}_{\mathrm{i}} \cdot \mathrm{S}_{\mathrm{j}}$ scatter the electrons. Langer and Fisher found that there are two sorts of resistive anomalies at a ferromagnetic transition: for $\mathrm{T}>\mathrm{T}_{\mathrm{c}}$, $\mathrm{d} \rho / \mathrm{dT} \sim\left(\mathrm{T}-\mathrm{T}_{\mathrm{c}}\right)^{-\alpha}$ where $\alpha$ is the specific heat exponent (which is believed to be slightly negative for the three dimensional Heisenberg model) while for $\mathrm{T}<\mathrm{T}_{\mathrm{c}}$ there is an additional contribution to the resistivity proportional to the square of the magnetization. These general conclusions apply also to the present model. 
One important consequence of the difference in models is that the peak at $\mathrm{T}>\mathrm{T}_{\mathrm{c}}$ found for small $\mathrm{k}_{\mathrm{F}}$ by Langer and Fisher is absent in the present model. The point is that the function $\mathrm{B}$ has range $\mathrm{k}_{\mathrm{F}}^{-1}$. In the range where this is greater than the magnetic correlation length, the two spin correlator in the Langer-Fisher expression for $\rho$ diverges in the same way as the uniform susceptibility, i.e. as $\xi^{4}$ In the present case, the quantity in square brackets is the long-wavelength fluctuations in $S_{i} \cdot S_{j}$, which has the weaker divergence $\xi^{4-\mathrm{d}-2 \eta}$. In the calculations we have performed for $0.5 \leq \mathrm{k}_{\mathrm{F}} \mathrm{a} \leq 1$ the divergence is not visible.

For $\mathrm{T}<\mathrm{T}_{\mathrm{c}}$, or in a magnetic field the same formula applies except that one must distinguish between longitudinal and transverse fluctuations, and one must add terms in which two of the spins in Eq. 11 are replaced by the uniform magnetization. These terms lead to a contribution to $\rho$ proportional to $\mathrm{M}^{2}$ which is absent in the model of Langer and Fisher. In particular, in the model of Langer and Fisher, the only effect of a nonzero $\mathrm{M}$ was to decrease the total amplitude of the spin fluctuations, leading to a decrease in $\rho$ below $T_{c}$. In the present model, this effect competes with the four additional scattering terms of form $\mathrm{M}^{2}\langle\mathrm{~S}(0) \cdot \mathrm{S}(\mathrm{R})\rangle$ which lead to an increase in the resistivity.

We have evaluated Eq (11) using Eq. 12 for B and calculating the spin correlator in the spherical model. Results are shown in Fig. 1 for $\mathrm{p}_{\mathrm{F}} \mathrm{a}=1$ and two magnetic fields: $\mathrm{H}=0$ and $\mathrm{H}=0.1 \mathrm{~T}_{\mathrm{c}}$. Results for $\mathrm{p}_{\mathrm{F}} \mathrm{a}=0.5$ are very similar. Note that the results are consistent with the predictions of Langer and Fisher: in the spherical model $\alpha=-1$ so $\mathrm{C}$ and $\mathrm{d} \rho / \mathrm{dT}$ have derivative discontinuities at $\mathrm{T}_{\mathrm{c}}$ and below $\mathrm{T}_{\mathrm{c}}$ an additional term, proportional to $\mathrm{M}^{2}$, is operative. However, in the spherical model approximation used here the sign of this term is positive: the resistivity increases below $\mathrm{T}_{\mathrm{c}}$ or in a field.

The resistivity implied by Eq. 6 has been previously calculated by Kubo and Ohata [3], Searle and Wang [2], and Furukawa [4]. Searle and Wang and Furukawa used mean field approximations in which all spin correlations are neglected, i.e. $\left\langle\mathrm{S}_{\mathrm{i}}\right.$. 
$\left.\mathrm{S}_{\mathrm{j}}\right\rangle-\left\langle\mathrm{S}_{\mathrm{i}}\right\rangle\left\langle\mathrm{S}_{\mathrm{j}}\right\rangle=0$. It is evident from the previous discussion that these correlations are essential. Furukawa used an "infinite dimensional" approximation in which he found that for $\mathrm{T}>\mathrm{T}_{\mathrm{c}}$ the core spins fluctuated very rapidly (i.e. on the scale set by $\mathrm{t}$ ) and led to an enormous imaginary part $\left(\approx \mathrm{J}_{\mathrm{H}}\right)$ to the electron self energy. It is difficult to reconcile these results with those presented here. In our work $J_{H}$ drops out of the problem and the $S_{i}$ are seen to be well described near $T_{c}$ by a classical Heisenberg model which entails fluctuation rates of order $\mathrm{T}$ or less. Kubo and Ohata obtained via a different method an expression very similar to our Eq. 18, but evaluated the spin correlation function using an approximation which neglected the fluctuations in $\left\langle\mathrm{S}_{\mathrm{i}} \cdot \mathrm{S}_{\mathrm{j}}\right\rangle$ which are responsible for the up-turn we find in $\rho$ near $\mathrm{T}_{\mathrm{c}}$.

Some representative experimental data from ref [16] are shown in the inset to Fig. 1. Similar experimental results have been obtained by many authors [1,2, 17, 18]. Although the qualitative temperature dependence calculated for $\mathrm{T}>\mathrm{T}_{\mathrm{c}}$ is consistent with the data several important discrepancies are evident: the calculated resistivity has the wrong magnitude (by several orders of magnitude), a far too weak doping dependence, and an incorrect behavior for $\mathrm{T}<\mathrm{T}_{\mathrm{c}}$ or in a field. Some of these discrepancies may be due to the inadequacy of the spherical model, but the magnitude and doping dependence cannot easily be explained away. The results may be traced to the fact, evident already in Eqs. 9 and 10, that in the double exchange model the magnetic fluctuations are a weak perturbation on the electron hopping and imply a $\mathrm{k}_{\mathrm{F}} \ell \gg 1$.

The discrepancy suggests that some other mechanism, not present in the double exchange model, must act to substantially reduce the electron hopping. We suggest that this mechanism is a polaron effect due to a very strong electron-phonon coupling stemming from a Jahn-Teller splitting of the $\mathrm{Mn}^{3+}$ ion. Other authors, most notably Kusters et. al. [17 have argued in favor of a magnetic polaron picture. Our calculation shows that the standard double exchange Hamiltonian does not contain magnetic polaron effects because the effective carrier-spin interaction is too weak to lead to the 
formation of polarons. On the other hand, the Jahn-Teller coupling is very strong. It causes the cubic-tetragonal transition observed at $\mathrm{T}^{*} \approx 800 \mathrm{~K}$ in $\mathrm{LaMnO}_{3}$ [6], and in fact, $\mathrm{T}^{*}$ is a dramatic underestimate of the basic Jahn-Teller energy. Using the standard Jahn-Teller Hamiltonian and the measured [19] oxygen displacements one finds that this energy is $\sim 1 \mathrm{eV}$, much greater than the measured electron kinetic energy at $\mathrm{x}=0.175$. It therefore seems likely that the Jahn-Teller energy remains important even in the metallic regime $0.2<\mathrm{x}<.45$. In this picture the physics would involve a crossover between a high $\mathrm{T}$, polaron dominated disordered regime and a low $\mathrm{T}$ metallic ordered regime. Understanding this crossover requires a theory of the interplay of polaron and metallic physics which is beyond the scope of this paper. However, it is clear that if polaron physics reduces the mean free path to less than a lattice constant, the argument previously given that the carrier resistivity is independent of the mass does not apply. Mathematically, if the electron self-energy is very large, than the hopping part of the electron Green function is proportional to $\mathrm{t}$, not $1 / \mathrm{t}$ and so the memory function, Eq. (11) scales as $\mathrm{t}^{(4)}$, not $\mathrm{t}^{(0)}$. Then the increase in velocity for $\mathrm{T}<\mathrm{T}_{\mathrm{c}}$ or $\mathrm{H} \neq 0$ will compete with the extra scattering terms and may lead to drop in resistivity for $\mathrm{T}<\mathrm{T}_{\mathrm{c}}$, as observed.

In conclusion, we have presented and compared to data a solution of the "double exchange" Hamiltonian widely believed to describe the physics of $\mathrm{La}_{1-x} \mathrm{Sr}_{\mathrm{x}} \mathrm{MnO}_{3}$. We noted the existence of a relation between an optical property (the low frequency spectral weight) and a magnetic property (the spin-wave stiffness). Experimental measurements of the spin wave stiffness would be a useful test of the model. We showed that the calculated resistivity is much too small, and has an incorrect field and temperature dependence, and we proposed that polaron effects are responsible for the discrepancy. 


\section{ACKNOWLEDGEMENTS}

We thank Dr. D. E. Cox for drawing our attention to ref [19] and Dr. P. Schiffer

for many interesting discussions of the data and for drawing our attention to ref [17. 


\section{REFERENCES}

[1] S. Jin, T. H. Tiefel, M. McCormack, R. A. Fastnacht, R. Ramesh and L. H. Chen, Science 264, 413 (1994).

[2] C. W. Searle and S. T. Wang, Canadian Journal of Physics 48, 2023 (1970).

[3] K. Kubo and N. Ohata, J. Phys. Soc. Jpn. 33, 21 (1972).

[4] N. Furukawa, J. Phys. Soc. Jpn, in press.

[5] C. Zener, Phys. Rev. 82, 403 (1951), P. W. Anderson and H. Hasegawa, Phys. Rev. 100, 675 (1955), P. G. deGennes, Phys. Rev. 118, 141 (1960).

[6] J. Goodenough, Phys. Rev. 100, 564 (1955).

[7] C. Herring, Magnetism, vol 2, J. Rado and H. Suhl, eds.

[8] L.F. Mattheiss, private communication.

[9] C. Kittel Quantum Theory of Solids (Wiley, New York) 1963 Eq. 26 of Ch. 2.

[10] G. S. Rushbrooke, G. A. Baker, Jr. and P. J. Wood in Phase Transitions and Critical Phenomena C. Domb and M. S. Green, eds., (Academic Press: New York) 1974. See especially eq 5.4.

[11] A. J. Millis and S. N. Coppersmith, Phys. Rev. B42i 10807 (1990).

[12] T. Arima, unpublished.

[13] J. M. Ziman Models of Disorder.

[14] W. Gotze and P. Wolfe, Phys. Rev. B6, 1226 (1972).

[15] M. E. Fisher and J. S. Langer, Phys. Rev. Lett. 20, 665 (1968).

[16] Y. Tokura, A. Urushibara, Y. Moritomo, T. Arima, A. Asamitsu, G. Kido and N. Furukawa, unpublished. 
[17] R. M. Kusters, J. Singleton, D. A. Keen, R. McGreevy and W. Hayes, Physica B155 p. 362 (1989).

[18] P. Schiffer, A. P. Ramirez, W. Bao and S-W. Cheong, unpublished.

[19] J. B. A. A. Elemans, B. vanLaar, K. R. vanderVeer and B. O. Loopstra, J. Sol. St. Chem. 3, p.238 (1971). 


\section{FIGURES}

FIG. 1. Resistivity calculated from double exchange model as described in the text.

The solid line is the resistivity in zero field; the dashed line is the resistivity in a field of $0.15 \mathrm{~T}_{\mathrm{c}}$. The inset displays data from Tokura et. al.. 